\title{
Wave Nature of Fractal Lacunarity: A Higher Resolution Scale to Capture Subtle Changes in ECG Signals
}

\author{
AN Esgiar, PK Chakravorty \\ University of Al Tahadi, Sirte, Libya
}

\begin{abstract}
Lacunarity is a feature of a fractal set. Its wave nature was predicted theoretically by Bloomenfeld and Mandelbrot. In this paper, we present our result confirming the existence of wave nature of lacunarity for ECG signals which are statistical fractal sets. We have applied this wave nature of lacunarity to capture the subtle changes in ECG signals in a very short time span of about one minute to predict occurrence of termination of atrial fibrillation for a subject, whose ECG is being monitored continuously. This method can be used to detect the minute subtle changes of ECG signals which are caused by other physiological events as well and needs further investigation to standardize it .
\end{abstract}

\section{Introduction}

The ECG signals are statistical fractal sets. Lacunarities are measures of structure in a fractal set i.e., distribution of gaps in a point set. Lacunarities are more important in classification or segmentation than other fractal features like fractal dimension and mass dimension. This was shown for ECG signals in our paper presented in 2004 conference[1].

It is known that if an $\varepsilon$-ball of radius $\Re$ is placed on each point of a fractal set then the total mass measure is;

$$
M(\Re)=F \Re^{\delta}
$$

Blumenfeld and Mandelbrot [2] have argued that if the error of best linear fit in the data $\left\{\log (\Re),-\log \left(M_{\text {ball }}(\Re)\right)\right\}$ is zero then the set is random fractal and the structure is self-similar at all scales of reduction i.e., distribution of the prefactor $F$ in equation- 1 is independent of $\Re$. Further this prefactor $F$ represents the lacunarity measure of the set. A systematic fractal such as the Cantor dust is self similar with respect to a reduction ratio $r$ that fall into a geometric sequence and if an $\varepsilon$-ball of radius $\Re$ is placed on each point of the set then for such sets distribution of lacunarity with respect to $\Re$ is a noisy wave and roughly has a period of $r$.
ECG signal is a statistically fractal set (unlike any systematic fractal set) and hence its lacunarity measure is a function of the box size and it can be proved that the lacunarity (based on even moments of mass) plot should be generally convex upwards and lacunarity (based on odd moments of mass) should be generally concave upwards as was presented earlier[1]. In this paper, first we confirm the prediction of Bloomenfeld and Mandelbrot regarding the wave nature of Lacunarity. This wave nature of lacunarity is used to discern the changes occurring in the ECG signals in a very short time span.

All the fractal features have been estimated by a new box counting algorithm. We have named it True Box Counting method. This algorithm estimates the fractal features like fractal dimension, mass dimension and lacunarities based on different moments of mass accurately and reliably.

\section{Methods}

The atrial fibrillation ECG signals data set files with their annotation files were obtained from Physionet challenge 2004 [3]-learning sets S \& T. These data sets contains ECG signals collected from ten subjects (persons). For each subject two signals had been collected. We called them signal1 and signal2. The set $S$ has ECG signals of 10 subjects which were collected one minute before the termination of atrial fibrillation. The set $T$ has ECG signals of the same 10 subjects and were collected just before the termination of atrial fibrillation.

\subsection{Estimation of fractal features}

An ECG signal is a statistically fractal set $(y=f(x))$ and constituted a point set in $E^{2}$ space. The fractal feature of this point set was estimated by True Box Counting algorithm described in paper presented earlier [1].

- Lacunarity based on $2^{\text {nd }}$ mass moments was estimated as proposed by Mandelbrot[4, 2].

$$
\Delta(L)_{2}=\frac{M_{b o x}^{2}(L)}{\left(M_{b o x}(L)\right)^{2}}-1
$$




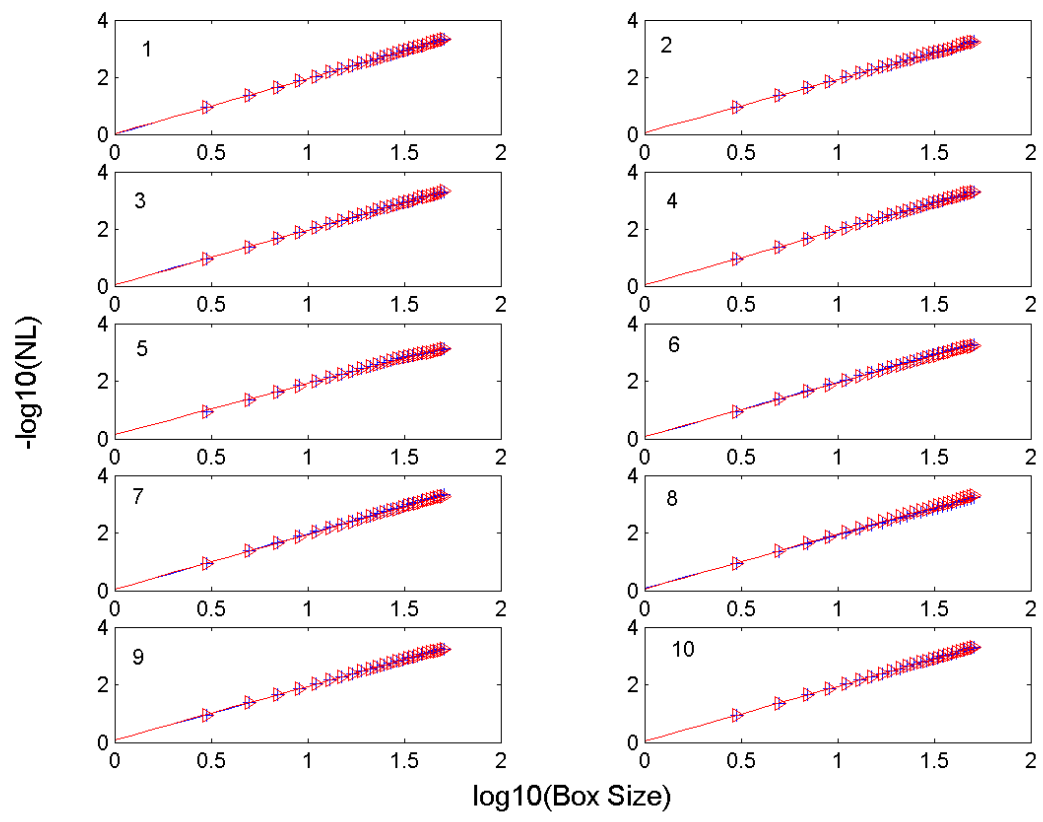

Figure 1. Fractal dimension plot - slope of $\left\{\log (L),-\log \left(N_{\text {box }}(L)\right)\right\}$ - for all subjects. blue + is from set $\mathrm{S}$, signal-2 and red $\triangleright$ is from set $\mathrm{T}$, signal-2.
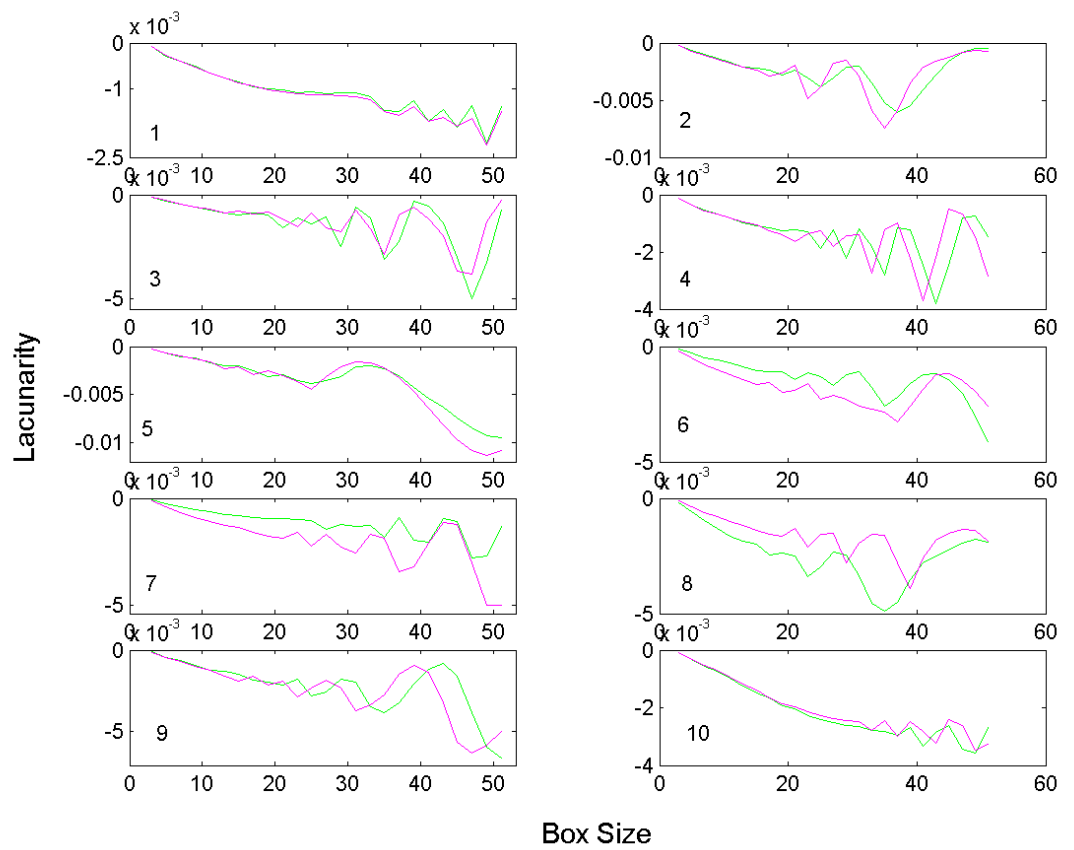

Figure 2. Lacunarity based on $15^{\text {th }}\left\{\Delta(L)_{15}-\mathrm{L}\right\}$ plot for all subjects. Green is from set S, signal-2 and Magenta is from set T, signal-2. 
Similarly, lacunarity based on higher mass moments were evaluated.

- Lacunarity based on $3^{r d}$ mass moment:

$$
\Delta(L)_{3}=\frac{M_{b o x}^{3}(L)}{\left(M_{b o x}(L)\right)^{3}}-3 * \frac{M_{b o x}^{2}(L)}{\left(M_{b o x}(L)\right)^{2}}+2
$$

The number of boxes required to span the point set $N_{\text {box }}(L)$ - and various mass moments - $M_{b o x}^{q}(L)$ - were estimated up to box size 51, i.e, $\mathrm{L}=51$.

For perfectly fractal sets like Cantor set, Sierpinski triangle, Sierpinski curve the lacunarity plot $\left(\Delta(L)_{n}\right.$ Vs. L) yields a line parallel to abscissa. For a statistically fractal set, lacunarity $\Delta(L)_{n}$ is a function of box size, 'L'. For such a curve, we used the power law to best fit the data points.

- The equation for best fit:

$$
\Delta(L)_{n}=\left(a_{n}\right) *(L)^{m_{n}}-1
$$

For perfectly fractal sets the value of index $m_{n}$ is zero and coefficient $a_{n}$ represented the lacunarity of the set.

\subsection{Selection of ECG signals for analysis}

The lacunarity Vs. box size plots $\left\{\Delta(L)_{n}-\mathrm{L}\right\}$ for each subject from set $S$ and $T$ were plotted on the same axes for signal-1 and signal-2 separately (figure- 2 presents the plot for signal-2, the lacunarity plots for signal-1 is not presented due to lack of space). It was seen that plots were wavy as predicted by Bloomenfeld and mandelbrot. Further, it was seen visually that in lacunarity plots for signal-2, the difference in frequency between the set $\mathrm{S}$ data and set $\mathrm{T}$ data were more for all the subjects. Thus signal-2 was selected for the analysis of the result.

\subsection{Qualitative measurement of frequency difference}

The difference in frequency between lacunarity plot of S-type signal and T-type signal was determined in the following two steps.

1. At first the normalized cross-correlation function $x$ corr in Matlab was used to estimate the phase difference between these two (i.e., S-type and T-type lacunarity) sequences. It yields a plot of phase lags between these two sequences.

2. This plot was further analysed to estimate of the frequency difference between these two sequences.

The frequency difference between S-type and T-type signal (for signal-2) was estimated by this method.

\section{Results}

The fractal dimension plot - slope of $\{\log (L)$, $\left.\log \left(N_{\text {box }}(L)\right)\right\}$ - is presented in figure-1 for signal-2. Each

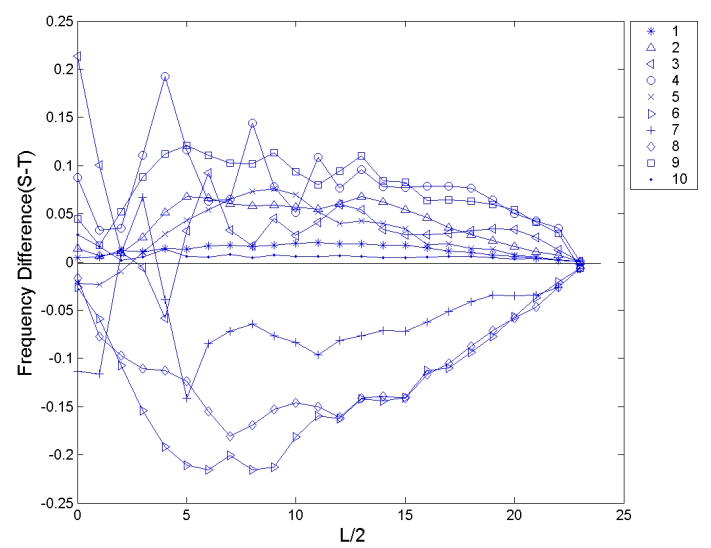

Figure 3. The change in frequency plot between $\mathrm{S}$ and $\mathrm{T}$ signals (signal-2) with the total sequence length. Legends are for subject 01 to subject 10

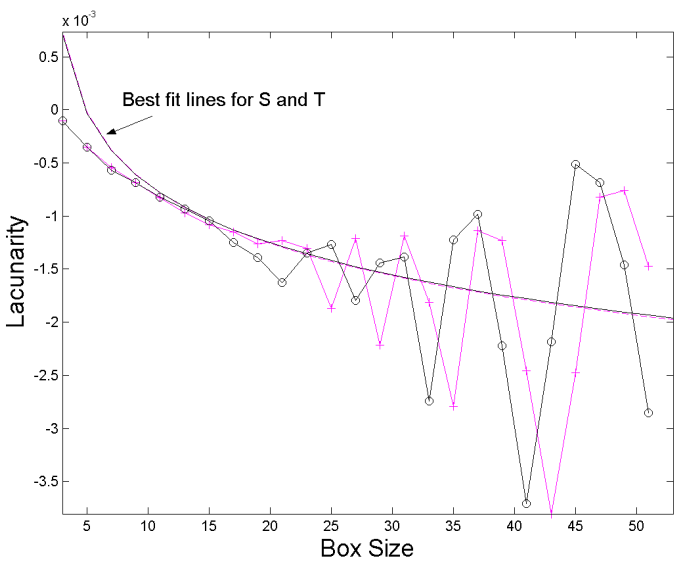

Figure 4. The lacunarity plot with the best fit lines for S (symbol + ) and $\mathrm{T}$ (symbol o) signals (signal-2). The best fit line magenta is for $\mathrm{S}$ and black is for T. Both the best fit lines have merged. This is for subject- 4

sub-image in this figure corresponds to one subject and contains fractal dimension plot of signal-2 from set $S$ and set $T$.

The lacunarity plot -(based on $15^{\text {th }}$ moment of mass, $\left.\left\{\Delta(L)_{15}-\mathrm{L}\right\}\right)$ - is presented in Fig.2 for signal-2. Each sub-image in this figure corresponds to one subject and contains lacunarity plot of signal-2 from set $S$ and set $T$.

The difference in frequency between $\mathrm{S}$ and $\mathrm{T}$ type signal is presented in figure-3. The legend corresponds to subjects 01 to 10 .

The lacunarity plots for subject-4 and subject-6 are presented in figure- $4 \& 5$. The best fit lines obtained by equation- 3 are superimposed on them. 


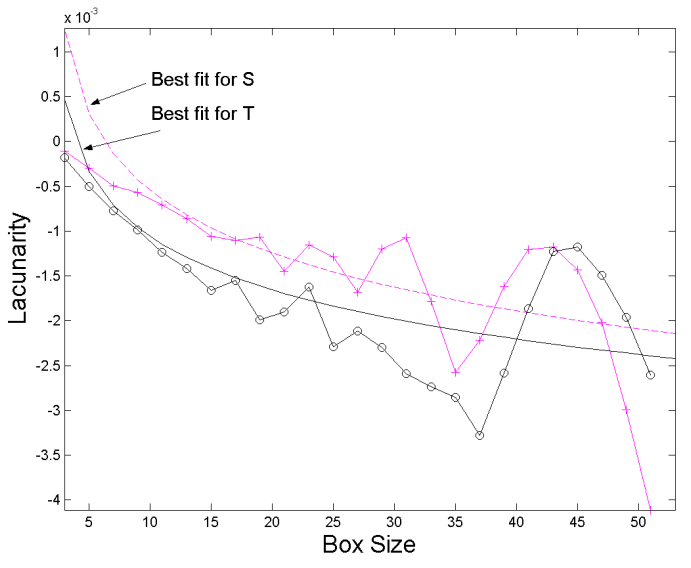

Figure 5. The lacunarity plot with the best fit lines for S (symbol + ) and $\mathrm{T}$ (symbol $\mathrm{o}$ ) signals (signal-2). The best fit line magenta is for $\mathrm{S}$ and black is for T. This is for subject- 6

\section{Discussion and conclusions}

The ECG data sets $\mathrm{S}$ and $\mathrm{T}$ for each subject was collected within one minute difference. Thus it is likely that they are quite similar to each other. The fractal dimension plot shown in figure-1 fails to show the difference between the $\mathrm{S}$ and $\mathrm{T}$ signals for each subject, as the fractal dimension, i.e., the slope of of the best fitting lines are effectively same for all the subjects.

The lacunarity plots based on different moments of mass showed wavy nature. But as the mass moments were increased the frequency difference (between lacunarity of $\mathrm{S}$ and $\mathrm{T}$ type data set) curves presented in figure3 showed much clarity. Thus the the lacunarity based on higher moment of mass yields more information, this was also proved statistically in the paper presented in last conference[1]. This wave nature of lacunarity was predicted by Bloomenfeld and Mandelbrot [2] earlier basing it on logic. Lacunarity curves obtained by applying TBC algorithm proves their theoretical prediction. This conversely also proves the accuracy of TBC in estimating the fractal features as to our knowledge, no other algorithm has been able to obtain these curves.

The frequency of lacunarity waves for S-type signal is higher than T-type signal for subject 01, 02, 03, 04, 05, 09 and, 10. This is shown in figure- 3 also. This difference in the frequency is possibly due to difference in termination of atrial fibrillation and needs more cardiologic investigations. The amplitudes of the lacunarity wave varies between subjects as they perhaps belong to different state of physiological state and age.

For all cases, a finite differnce in lacunarity (the best fit lines obtained by equation-3) was observed (as presented in figure-5), excepting for subject-4, refer figure-4. For subject-4, the lacunarity estimated by the equation-3 for $S$ and $T$ - type Signals, shown in figure-4 have the same value but a careful examination reveals the difference in frequency in the lacunarity wave, which is actually a more fundamental measure than that of estimates obtained from equation-3.

Thus it is possible to have two point sets which have identical or near identical fractal dimension estimated by the slope of the best fitting line for the points $\{\log (L)$, - $\left.\log \left(N_{\text {box }}(L)\right)\right\}$ as well as identical or near identical lacunarity estimated by equation-3. The wave nature and the frequency of the lacunarity wave is the only feature which distinguishes between such sets. This change in frequency actually refers to change in the reduction ratio of similarity of a set.

Mathematically it can be shown (due to space limitation we are not showing it here) that the cases like subject- 4 are not isolated ones. It is rather possible to have many such sets, where fractal dimension and lacunarity estimates may be same or similar but the frequency of the lacunarity wave for such sets shall be different from each other.

It may be conjectured based on this limited study that the fractal nature of sets (signals or images) representing biological processes evolve first with a change in frequency of lacunarity and then other feature of the set changes with time. Thus the wave nature of lacunarity especially based on higher moments are important tools in classification of biological signals or images representing such evolutionary processes.

\section{Acknowledgements}

The authors gratefully acknowledge the active support provided by the faculty of engineering, Al Tahadi University, Sirte, Libya in carrying out this research work. We also acknowledge the efforts of Challange team of www.physionet.org family for supplying us with high quality data sets to test, prove and, present our methods. It was otherwise difficult and perhaps our ideas would have remained on our desks only.

\section{References}

[1] Esgiar A, Chakravorty P. Electrocardiogram signal classification based on fractal features. In Murray A (ed.), Computers in Cardiology. IEEE, 2004; .

[2] Blumenfeld R, Mandelbrot BB. Levy dusts, mittag-leffler statistics, mass fractal lacunarity, and perceived dimension. Physical Review E 1997;56:112-118.

[3] Physionet/computers in cardiology challenges February 2004;Downloaded from internet, http://www.physionet.org/challenge/.

[4] Voss RF. Random fractals: Characterization and measurement. In R.Pynn, A.Skjeltorp (eds.), Scaling Phenomena in disord. systems. Plenum, New York, 1986; . 